\title{
ARTICLE
}

\section{Risk assessment and management in obsessive-compulsive disorder}

\author{
David Veale, Mark Freeston, Georgina Krebs, Isobel Heyman \& Paul Salkovskis
}

David Veale is an honorary senior lecturer and a consultant psychiatrist in cognitive behaviour therapy at the NIHR Biomedical Research Centre for Mental Health, The South London and Maudsley NHS Foundation Trust, King's College London, and The Priory Hospital North London. Mark Freeston is Professor of Clinical Psychology at Newcastle University, Newcastle upon Tyne. Georgina Krebs is a clinical psychologist working in the service for young people with obsessive-compulsive disorder at the Maudsley Hospital, London. Isobel Heyman is an honorary senior lecturer at the Institute of Psychiatry, King's College London, and a consultant child and adolescent psychiatrist working in the service for young people with obsessive-compulsive disorder at the Maudsley Hospital, London. Paul Salkovskis is

Professor of Clinical Psychology and Applied Science at the Institute of Psychiatry and Clinical Director of the Maudsley Hospital Centre for Anxiety Disorders and Trauma. Correspondence Dr David Veale Centre for Anxiety Disorders and Trauma, Maudsley Hospital, 99 Denmark Hill, London SE5 8AF, UK. Email: David.Veale@iop.kcl.ac.uk

\begin{abstract}
SUMMARY
Some people with obsessive-compulsive disorder (OCD) experience recurrent intrusive sexual, aggressive or death-related thoughts and as a result may be subjected to lengthy or inappropriate risk assessments. These apparent 'primary' risks can be dealt with relatively easily through a careful understanding of the disorder's phenomenology. However, there are other, less obvious 'secondary' risks, which require more careful consideration. This article discusses the differentiation of intrusive thoughts and urges in people with OCD from those experienced by sexual or violent offenders; assessing the risk of self-harm and suicide; discussing the nature of repugnant obsessions with a patient; assessing risk of harm and violence to the dependents and family living with someone with the disorder; and assessing the lack of insight and the use of the Mental Health Act. Issues specifically related to children and young people with OCD are also highlighted.
\end{abstract}

\section{DECLARATION OF INTEREST}

None.

One of the recommendations of the National Institute for Health and Clinical Excellence (NICE) (National Collaborating Centre for Mental Health 2005: p. 15) guidelines on obsessive-compulsive disorder (OCD) is:

If healthcare professionals are uncertain about the risks associated with intrusive sexual, aggressive or death-related thoughts reported by people with OCD, they should consult mental health professionals with specific expertise in the assessment and management of OCD. These themes are common in people with OCD at any age, and are often misinterpreted as indicating risk.

Our article discusses issues to be considered when conducting a risk assessment in a person with intrusive sexual, aggressive or death-related thoughts and contrasts the phenomenology of such thoughts in OCD with that of similar thoughts in people who are at risk of sexual or violent offending or self-harm. It discusses the initial management of repugnant obsessions and pitfalls that may occur.

Professionals presented with an individual describing thoughts of violence, suicide or causing harm to children may be tempted to play for safety when conducting a risk assessment. However, a person with OCD can be harmed by an incorrect or unduly lengthy risk assessment, responding with increased doubts and fears about the implications of their intrusive thoughts. At best this will lead to greater distress, avoidance and compulsive behaviours, and mistrust of health professionals; at worst, to complete decompensation of the patient or break-up of the family. In reality, there is no need for overcautious reactions. Provided the clinician has appropriate expertise in OCD, there are very rarely any serious doubts about the diagnosis.

Another recommendation of the NICE guidelines (National Collaborating Centre for Mental Health 2005: pp. 14-15) is:

In people who have been diagnosed with OCD, healthcare professionals should assess the risk of self-harm and suicide, especially if they have also been diagnosed with depression. Part of the risk assessment should include the impact of their compulsive behaviours on themselves or others.

This article therefore discusses assessing the risk of harm to self and others from compulsions and comorbidity, risk in therapy, poor insight and use of the Mental Health Act.

\section{Phenomenology of obsessions and compulsions}

Risk assessment in OCD requires that the clinician has a good working knowledge of the phenomenology of the disorder. Obsessions are unwanted intrusive thoughts, doubts, images or impulses that repeatedly enter the mind ('intrusions'). They are distressing and ego-dystonic, but are acknowledged by the patient as unreasonable or excessive. A minority have overvalued ideas, which can lead to confusion with psychosis (Veale 2002). Compulsions are repetitive behaviours or mental acts that the person feels driven to perform. They can either be overt and observed by others (e.g. checking that a door is locked) or covert mental acts that cannot be observed (e.g. mentally repeating a certain phrase).

Intrusive thoughts, doubts or images are almost universal in the general population and their content is indistinguishable from clinical obsessions 
(Rachman 1978). By definition, such intrusions occur outside the volition of the person experiencing them, and tend to interrupt other mental activity because they are inconsistent with the person's usual value system. Examples of normal intrusive thoughts include the image of touching the genitalia of a child, worry (in the absence of any evidence) as to whether one had caused an accident on the way home, urges or impulses to attack a loved one with a kitchen knife, or thoughts and images of jumping onto a railway track in front of an oncoming train. Because these types of intrusion can and do feature in OCD, the difference between normal intrusive thoughts and obsessional thoughts lies not in the content itself but in the appraisals that individuals with the disorder attach to the occurrence or content of the intrusions (Salkovskis 1985, 2008; Rachman 1997). These appraisals and the compulsions that they motivate are thought to determine why intrusions are more frequent and distressing in OCD.

\section{Risks in OCD}

The phenomenology of OCD should lead us to consider two types of risk (Key points 1). The first primary risk - is only apparent and arises directly from an obsession. The risk is that the patient will act on an obsession (e.g. suicide or sexual acts with a child) or impulsively act out an obsessional fear. At its simplest, this need never be a concern: there are no recorded cases of a person with OCD carrying out their obsession. By definition, such intrusions are unacceptable and ego-dystonic, and the person is no more likely to act on their intrusions than a person with height phobia is to jump off a tall building. The obsession represents a type of fear or worry that the patient does not want to happen; like all fears or worries, it concerns ideas that the patient wishes to avert at all costs.

Of much greater concern is secondary risk - the unintended consequence of acting on compulsions and urges to avoid anxiety-provoking situations. There can be significant secondary risk associated with OCD. For example, a person with contamination fears in relation to eating and drinking may severely restrict food and fluid intake, leading in some cases to dehydration, malnourishment,

\section{KEY POINTS 1 Risk in OCD}

- Primary risk Risk arising directly from an obsession

- Secondary risk Unintended consequences of acting on compulsions and urges to avoid anxiety-provoking situations and in children growth retardation and arrested pubertal development. Another example may be harm from overexposure to disinfectant following from a compulsion to clean. By analogy, this is like someone with a phobia of wasps who drives off the road when they realise that there is a wasp in the car. Secondary risks also occur from compulsive hoarding, ${ }^{\dagger}$ when a house may become unsanitary, damp or a fire risk as a result of the behaviour.

More commonly, irreversible harm may occur in young people whose OCD is severe during critical phases of development. For example, withdrawal from school and social interactions because of severe OCD can lead to significant deficits in cognitive and social development. Individuals may fail to develop autonomy in key areas owing to their inability to carry out normal routines and their dependence on others. All people with the disorder find that symptoms frequently interfere significantly with life opportunities, with some chances lost irretrievably.

Where compulsions are concerned, urges that are themselves obsessions need to be distinguished from urges arising as responses to obsessions. Once this is done, the risks are relatively obvious. Thus, if someone has an obsessional fear of cutting themselves, you can be very certain that they will not harm themselves. However, if the idea of harming others is the obsession, they might respond by cutting themselves rather than harming someone they love. Secondary risks are often subtle. For example, a parent who is constantly preoccupied with their obsessions may become less responsive and emotionally available to their children.

We will now apply these general principles to the assessment of specific concerns, first where there are intrusive sexual thoughts and then in the case of violent thoughts.

\section{Assessing intrusive sexual thoughts}

An assessment in OCD primarily involves clearly formulating the problems the patient is experiencing rather than seeking to 'rule out' forensic and sexoffending factors. Gordon \& Grubin (2004) have described how to assess risk in sexual offenders and Marshall \& Langton (2005) discuss the nature of sexual thoughts in sexual offenders. Box 1 outlines factors that might distinguish between sexual thoughts in OCD and in potential sexual offending. These are discussed in more detail below.

People who have OCD might worry about their intrusive sexual thoughts, images or impulsive urges, which are experienced as ego-dystonic and repugnant to the individual. They may be experiencing:
'See Lopez Gaston et al: Hoarding behaviour: building up the 'R factor'. pp. 344-53, this issue. Ed. 
BOX 1 OCD or potential sexual offender?

Various factors differentiate the intrusive sexual thoughts of people with OCD from those of sexual offenders

- Ego-dystonicity of the thoughts

- Failure to act on or masturbate to the thoughts

- Avoidance of trigger situations

- Efforts to suppress the thoughts

- Very frequent or constant occurrence of the thoughts

- Dominant anxiety, distress and guilt about the thoughts

- Overdisclosure of irrelevant past sexual history

-Wanting help and seeking referral to mental health services

- Presence of additional obsessive-compulsive symptoms

- a doubt that they might have acted upon such urges in the past (usually leading to a compulsion of mental reviewing of past actions);

- a worry that they may act upon such urges in the future (usually leading to avoidance of situations that are regarded as risky, together with repeated reassurance seeking); in a young person with little or no sexual experience, such worries may be especially intense in relation to sexual orientation;

- a worry that they may be wrongly accused of acting on such urges (usually associated with not disclosing details or repeated seeking of reassurance);

- an evaluation that they are a bad person for having such thoughts and images (usually leading to attempts to control, remove, or atone for the thought).

The difficulties in identifying intrusive thoughts as being related to OCD usually occur when the assessor lacks experience with the disorder. This is especially the case when people report thoughts and worries about acting on sexual or violent urges. Problems may arise for occupational health staff when assessing people with OCD who work with children and report intrusive sexual thoughts. Problems can also arise because either the patient or assessor seeks reassurance in ways that increase doubt or anxiety in both parties (e.g. 'Are you 100\% confident that these are obsessional thoughts rather than something else?'). This represents a transmission of obsessional worries from the patient to the clinician.

We highlight below the most common issues in assessing intrusive sexual thoughts about children. It is to be noted that no single difference is sufficient to make a differential judgement; the overall pattern of differences is what counts.
Is the intrusive thought or image ego-dystonic?

In adults with OCD, intrusive sexual thoughts are ego-dystonic, i.e. they are entirely repugnant. They are totally at odds with the values of the individual and there is no aspect of them that is attractive or sexually stimulating (but see the section on sexual arousal below). Thus, someone with OCD with intrusive thoughts about having sex with children is likely to be extremely caring towards children. They are likely to: (a) already be a parent and/or working with children (or would very much like to be so involved in the future); and (b) evaluate themselves as 'bad' for having such thoughts.

Ego-dystonicity in young people, however, can be especially difficult to assess, as they are often still in the process of sexual development and maturation. They may therefore feel very confused as to whether a sexual thought is wanted or unwanted, permitted or deviant. It is not uncommon for young people to have sexual obsessions involving children, parents, siblings, animals or objects, which may be very confusing and distressing. In children with OCD, especially the younger ones, there may be more difficulty in eliciting ego-dystonicity (Geller 2006). The young child may lack the cognitive maturity to identify thoughts as unwanted, may think it is 'weird' to say so, or may feel that if they admit to wishing they did not have symptoms, they may be asked to stop ritualising, which they fear would be difficult and anxiety-provoking. So it is important to note that the absence of ego-dystonicity or insight does not exclude the diagnosis of OCD, especially in a child or adolescent. By contrast, a paedophile is likely to regard sexual thoughts as ego-syntonic: to be more ambivalent about having such thoughts and to find them attractive at least some of the time or to be cautious about revealing their true feelings.

Is the current behaviour consistent with the intrusive thoughts and images?

People with OCD neither act on their intrusive sexual thoughts nor fantasise or masturbate about them. They may, however, experience the unwanted intrusions when they masturbate to an adult fantasy or have sex, and become confused as to whether the thoughts are ego-dystonic or whether they wanted to have them. Very rarely, people with OCD might appear to act once on their obsessional urge but in a way that is not significantly harmful. Thus, a man with OCD said he once accessed child pornography to check whether he was aroused by it but typically reacted with repugnance. A woman with OCD described how she once touched her baby daughter's genitalia to check whether she 
would insert her finger. Men with OCD may very rarely masturbate to an adult fantasy before going into situations where they may be in contact with a child with the goal of reducing the likelihood of sexual arousal while there. Sexual offenders are more likely to use sadomasochistic or paedophilic pornography regularly for pleasure.

Does the person try to avoid situations or activities that trigger the intrusive sexual thoughts and images?

People with sexual obsessions that involve children typically avoid being alone or near young people. Avoidance behaviour may be quite subtle, particularly in children and adolescents, where parents or other authority figures may prevent gross avoidance. For example, the teenager with a sexual obsession about younger children may attend school, but avoid looking at younger children and wait to catch a later bus after the younger children have gone home. A paedophile is more likely to consciously seek out places where children play or find opportunities to be alone with them.

Does the person attempt to suppress, neutralise or distract themselves from intrusive sexual thoughts?

Individuals with OCD are more likely to try to avoid, neutralise or suppress repugnant thoughts. They may sometimes examine them in detail to check whether they truly find them repulsive. A potential paedophile may sometimes try to suppress or avoid intrusive thoughts but is more likely to engage with them.

How frequent are the intrusive thoughts and images?

People with OCD are likely to experience frequent intrusive thoughts that may dominate their mental life as they constantly monitor their thoughts for signs of danger and then try to suppress them. A person at risk of paedophilia is likely to have intrusive thoughts that are less frequent and which are more likely to be cued by specific triggers such as childcare activities. Some sexual offenders experience intrusive thoughts only when they are intoxicated. When the thoughts are frequent then a paedophile is more likely to be elaborating them in detailed fantasies.

\section{What is the dominant emotion?}

People with OCD seek help because they are extremely anxious and distressed by thoughts, images or urges. They will feel guilty simply because they have the thoughts even though they do not experience any pleasure from them. A potential paedophile or violent offender is less likely to be distressed by such thoughts or the distress is understood by the motivation to avoid being caught. They are more likely to experience pleasure from the thoughts, although some may also feel guilty or remorseful early in their 'career' if they have acted on the thoughts in any way.

Does the person experience sexual arousal with intrusive thoughts?

Self-reported sexual arousal is an unreliable indicator of sexual interest. Someone with OCD may be especially sensitive and may consequently overreport arousal (Warwick 1990). They may monitor and check the state of arousal in their genitalia. However, selective attention and feedback on the degree of arousal may increase both blood flow and level of arousal. In addition, the experience of anxiety may be confused with sexual arousal. As far we are aware, phallometric evidence of response to sexual stimuli has not been conducted in OCD, nor should it. However, it has been shown that anxiety increases phallometric indices in normal men. Many clinicians believe the opposite to be true, leading them to doubt whether or not a patient with an obsession has an anxiety disorder when they report physical signs of arousal; some male patients show clear signs of physical arousal in obsessional situations - this is not an indication of sexual interest, but of anxiety (Warwick 1990).

\section{Is there comorbidity?}

Comorbidity with Axis I or II disorders may be present in both OCD and sexual offenders. However, the issue becomes complex when a person with OCD (in one domain such as contamination) is, for example, sexually immature and might have paedophilic sexual fantasies (a different domain). Thus, an understanding of the psychopathology is crucial as in such an individual the sexual thoughts are not ego-dystonic.

\section{Does the person easily disclose material?}

People with OCD are usually extremely ashamed of their intrusive thoughts and often do not reveal the details (equally, a paedophile may not reveal the details of their thoughts because of their fear of being caught). An interview with someone with OCD therefore needs to be sensitively handled. If an individual is unable to disclose the details of their intrusions, then the interviewer might offer a multiple choice answer (e.g. 'I myself have had and talked to a lot of patients with intrusive thoughts or images that concern, for example, rape, homosexuality, sex with children, sex with a parent or an animal, or some thought or image that I find personally unacceptable. Do you experience any intrusive thoughts like these?'). 
Another problem in OCD is the overdisclosure of an irrelevant past sexual history and a search for past peccadilloes (e.g. reporting that as an adolescent they once looked through a window with a pair of binoculars to view a woman undressing) in an effort to find evidence that they may be a sexual offender. Individuals may also be excessively concerned about their own exploratory sexual behaviour with peers during childhood or adolescence. They may report events where they think they may have become aroused inappropriately. Overreporting may be a way of reassurance-seeking or neutralising anxiety.

Occasionally, children and adolescents with comorbid behavioural disorders disclose their obsessions in quite an aggressive and intrusive way to provoke distress in others.

A paedophile is less likely to reveal information but more likely to have a history of exploitation or abuse of others, or of sexual offences.

What is the motivation to seek help?

People with OCD are motivated to seek help because they regard their intrusive thoughts or images as repugnant and they are fearful of acting on them. They are extremely worried about the implications of their urges towards children and about acting in such a way.

A paedophile is more likely to be worried by the consequences of being caught. However, sometimes people with OCD are concerned that they may be incorrectly accused (e.g. because they left DNA in the vicinity of a crime, and that others will think they are a bad person and the complete opposite of what they are).

\section{What is the nature of the referral?}

People with OCD are likely to have sought referral because they are very worried about their thoughts. Occasionally they will be referred for specialist evaluation because they have revealed the content to others who have also interpreted the thoughts as a sign of risk. A person at risk of paedophilia is less likely to seek help voluntarily and is more likely to be referred by an external agency.

\section{Are other symptoms of OCD present?}

Many people with OCD who experience intrusive thoughts and images of a personally repugnant sexual content will also have obsessions and compulsive behaviours in other domains (e.g. washing, checking, order). Other obsessivecompulsive symptoms are probably less likely to occur in sexual offenders.
Is there a history of sexual abuse, or of accidental or deliberate exposure to sexually explicit material?

There are obvious risks to missing a history of sexual abuse in a distressed child, but equally there are risks to incorrectly assuming that a child or adolescent with intrusive sexual thoughts has been abused, when in fact they have OCD alone. Thus, some children with OCD have compulsions that involve their genitals, such as excessive washing, repeated touching or checking behaviours. In most cases, however, these thoughts and behaviours are part of the disorder, not signs of sexual abuse, and excessive questioning or investigations for child sexual abuse can do significant harm.

Although sexual abuse must always be considered in a child with sexual preoccupations and/or behaviours, the authors of one case series they felt confident in excluding sexual abuse (Healy 1991). None of the children had disclosed abuse, nor did they show affective change when discussing this possibility or seem burdened by a 'secret'. In addition, family relationships were good, unlike those commonly seen with intrafamilial abuse, and symptoms improved quickly when the children received appropriate treatment for obsessions.

In another case series, however, the authors report onset of OCD in two young children after sexual abuse or sex-play, and suggest that these experiences had become incorporated into obsessional content (Freeman 2000). Both children rapidly disclosed these events, so appropriate interventions were incorporated into the management plan.

Thus, it is appropriate that a clinician assessing a child with sexual obsessions considers the issue of child sexual abuse while remaining aware that excessive questioning may be harmful.

\section{Assessing intrusive thoughts of violence}

Intrusive thoughts and images of violence or aggression can be assessed in exactly the same way as the primary risks of intrusive sexual thoughts, and similar issues arise (Box 2).

BOX 2 Factors suggesting OCD in thoughts of violence

- Ego-dystonicity

- Absence of past behaviour consistent with the thought

- Presence of avoidance behaviour (e.g. avoidance of knives or sharp implements)

- Frequent thoughts

- High degree of distress

- Strong motivation to seek help 


\section{Postnatal OCD}

Images of stabbing are common; intrusive thoughts such as putting the baby in a saucepan or microwave can also occur. Mothers often experience intrusions about acting on urges to suffocate or stab their baby. Such thoughts are quite prevalent in mothers and there are several well-described case studies (e.g. Sichel 1993; Maina 1999). The differential diagnosis is with puerperal psychosis ${ }^{\dagger}$ (especially in the absence of a history of OCD). However, OCD is extremely unlikely to first occur in the context of a depressive psychosis. Conducting an incorrect or lengthy risk assessment or placing an adult with the mother at all times can be harmful as it can have the unwanted effect of increasing her doubts, reducing her confidence and leading to greater distress, avoidance and compulsive behaviours. In the absence of other psychotic features, such thoughts can be understood as an escalation of less unusual thoughts about causing harm. In such cases, reports of thoughts or images of violent content can cause well-meaning mental health professionals inappropriately to anticipate a risk of infanticide or harm to a child.

\section{Secondary-risk violence}

People with OCD are occasionally violent not because of obsessions about violence but because of extreme distress when someone either inadvertently or deliberately tries to prevent or terminate the compulsion or 'breaks the rules'. The secondary risk is that individuals can become violent or intimidating towards family members or health professionals (e.g. if someone 'contaminates' a clean object or says a word that is avoided, a person with OCD can feel extremely threatened). This is particularly likely to occur in children or adolescents because there is commonly a very high level of family involvement in rituals, which can lead to a great deal of frustration and anger in the home (Storch 2007).

Another group vulnerable to angry or violent outbursts are people with OCD in the context of an autism-spectrum disorder, especially when there are very rigid routines. Such individuals commonly have a poor understanding of their own and others' mental states (Baron-Cohen 2001); they may therefore have difficulty in empathising with the effect of their compulsions on others, may be unable to explain what they are experiencing, or may be unable to deal with the situation by expressing affect appropriately. In such cases, the individual is not acting on their obsession; rather they are acting in response to the distress, frustration and anger when they are thwarted in

acting on their compulsion or avoidance in their usual way. Substance misuse can increase this impulsivity.

\section{Case study: When seeking help makes things worse}

A patient with OCD was worried that he might be a paedophile. When his niece sat on his knee, he started to panic because he was worried that he was becoming aroused. He went to the toilet to check whether his penis was 'getting any bigger'.

He sought help and saw a psychiatrist. Concerned by the patient's doubts about whether he was aroused, she arranged a full risk assessment and informed Social Services, who met with the patient and his family. As a consequence, his OCD became worse because his fears that he might be a danger to the child were now apparently confirmed. His doubts increased and he felt unable to seek further help as he believed that treating the thoughts as symptoms of OCD might cause him to let down his guard and to act on them.

Individuals with OCD may undergo unnecessary, lengthy and damaging risk assessments, which make their disorder worse. The case study is an illustrative case history. A person with OCD hates uncertainty and the initial assessment is not an appropriate time to introduce patients to tolerating doubt (although it may be important to do so later in therapy when there is a good therapeutic alliance). Box 3 shows how patients have described assessments by health professionals.

\section{Management of repugnant obsessions}

There are only two evidence-based treatments for OCD: cognitive-behavioural therapy (CBT) (Veale 2007) and selective serotonin reuptake inhibitors (SSRIs) (National Collaborating Centre for Mental Health 2005). There may be a lengthy waiting list for CBT and if an SSRI has been prescribed then there is usually a delay before any potential benefit is gained from the medication. It

BOX 3 Examples of patients' comments regarding their assessments

'He gave me a differential diagnosis which made me panic as it increased my doubts about whether I did have OCD.'

'She said that, to be on the safe side, it would be better if I avoided working with children until I had received treatment.

'He said SSRIs might reduce my sexual urges so I assumed he must think there was a problem.'

'She said I was unlikely to act out any urges but she was still obliged to notify Social Services.' †'dentifying and caring for women at risk of puerperal psychosis will be discussed by lan Jones \& Sue Smith in the next issue of Advances. 
is therefore helpful for the clinician to be confident about the diagnosis and provide good rationale for the patient's experience of repugnant obsessions. When working with children or teenagers and their parents, it is important to ensure that parents are educated alongside the young person, as parents may fear, for example, that their child is growing up to become a paedophile or sexual offender. This only contributes to the child's anxiety and avoidance as, for example, they may be made to stop doing their previous baby-sitting job. It is important for people with OCD and their families to understand the nature of intrusive thoughts and professionals need to be very confident in informing them (Box 4).

\section{Assessing risk of thoughts of self-harm and suicide}

\section{Primary risk}

The obsessional thoughts of someone with OCD may concern self-harm or suicide. In this respect they are no different from intrusive thoughts of violence to others and similarly present an apparent primary risk. For example, a person fears that they may put their fingers in an electric socket or jump from a great height or take their own life (when they do not want to). In fact, most people with OCD would not want to die by suicide because of the harm that they would cause to others through doing so. In cases where OCD has been clearly established, and the thoughts about the fear of self-harm or suicide (e.g. acting impulsively) are ego-dystonic, then the case should be managed as for any other intrusion about violence.

\section{Secondary risk}

The assessment of the secondary suicide risk of self-harm becomes more complicated if, over time, the individual becomes more depressed and hopeless or has a comorbid personality disorder that is associated with impulsive behaviour. People with OCD are at greater risk of suicide than the general population. In the survey by Torres et al (2006), at least a quarter of people with OCD had attempted suicide at some time in their life. In such situations, it is usually helpful to establish the main problem as well as the motivation for ending life. Motivation arises from increased feelings of hopelessness about progress in overcoming the disorder or accessing therapy, or the individual's belief that they pose a real danger to others. They may then desire to escape from their intrusive thoughts and make plans to end their life.

In a small number of instances, patients may take their own life as a compulsive act. For example, a person who is terrified that they will harm their loved ones may take their own life in order to protect them; we are aware of several sad cases of this happening. Others may have comorbid borderline personality disorder and be self-harming as a way of regulating their mood and reducing their level of anxiety (e.g. a person with borderline personality disorder may self-harm as a way of reducing feelings of contamination).

\section{Compulsive self-harm}

Occasionally, self-harming may become part of a compulsion; for example, cutting, burning or hitting oneself as a form of neutralising an unacceptable thought or image. We have seen cases in which

BOX 4 Reassuring patients and families about intrusive thoughts

- Intrusive sexual and violent thoughts are normal and very common in the general population

Clinicians should explain that a person would be abnormal and lack any morals if he or she did not have such thoughts and might disclose their own. The reason why individuals with OCD have such thoughts more frequently is because they worry a great deal about harming others and try not to have such thoughts. Intrusive thoughts are part of being human and if they are upsetting, this indicates a person's values. Thus, religious people are upset by blasphemous thoughts, moral people are upset by thoughts of acting immorally, and so peace-loving, gentle people with high standards of behaviour and a strong sense of what is right and wrong are upset by sexual or violent thoughts.
- A person with OCD is at no greater risk of causing harm than is any other member of the public (they may even be at a lower risk)

A search on the database of the special (high-security) hospitals in England revealed no patients with sexual offences admitted with a diagnosis of $\mathrm{OCD}$ since records have begun. The collective experience of $\mathrm{OCD}$ specialists from around the world shows that obsessions about causing harm do not lead people to harm others.

- People with OCD should not avoid cues that may trigger their obsession or make them worse and they should try to resist the urge to rationalise their experience or repeatedly reassure themselves

Rationalising and reassuring are covert compulsions which maintain their obsessions.
Thus, a young mother with OCD can and should be left alone with her baby when using kitchen knives or other items that have been avoided due to her fear of causing harm. The man worried about stabbing his partner might be encouraged to sleep with a knife by his bedside; the woman worried about intrusive sexual thoughts should be playing with children in the way they normally do - the clinician should tell her that if they had children they would be happy to hire her as a baby-sitter.

- Showing physical signs of sexual arousal is not a sign of being a paedophile - anxiety, selective attention and monitoring arousal in one's genitalia can increase these signs

Patients may benefit from self-help books that discuss these issues in more detail (Veale 2005). 
severe harm, including self-blinding, occurred as a direct result of a compulsion. Merely telling the person that they are at risk because of their potentially or actually self-injurious compulsion is very unlikely to help on its own. Such individuals are usually carrying out compulsions to prevent themselves from being responsible for harm to others, so appealing to their sense of selfpreservation is ineffective.

\section{Comorbidity}

Comorbidity can complicate the assessment of risk in OCD and there may be risks related to the comorbid disorder. However, a good understanding of the phenomenology in the particular patient will resolve such issues.

\section{Depression}

Depression is the most common comorbid condition. It is often secondary to OCD, in which case:

- the patient describes their OCD as their biggest problem;

- the symptoms of depression developed after the onset of the OCD;

- the person predicts that the symptoms of depression would improve if the OCD were effectively treated.

Very occasionally patients have a comorbid bipolar disorder or recurrent depression in which obsessions and compulsions are symptoms of depression. Here the onset of depression and symptoms of OCD are simultaneous, and if the depression is effectively treated then the OCD improves.

The most important message to give to people with OCD who are suicidal is that the disorder is treatable. Behavioural activation is probably the most helpful psychological approach in OCD with comorbid depression. This is because if the depression is severe, it may be more effective than standard cognitive therapy (Dimidjan 2006; Veale 2008). It also targets cognitive processes such as depressive rumination and avoids the risk of patients trying to challenge the content of their obsessions, which is unhelpful. Sometimes it may take time to access a specialist service in CBT for OCD or to find the right approach. For pharmacotherapy of depression comorbid with OCD, potent or selective serotonin reuptake inhibitors are more effective than noradrenergic reuptake inhibitors (Hoehn-Saric 2000). If two or more selective serotonin reuptake inhibitors (SSRIs) are ineffective, then augmenting the SSRI with a noradrenergic reuptake inhibitor may be helpful (Mancini 2002).

\section{Schizophrenia and psychosis}

Obsessive-compulsive disorder can also be comorbid with schizophrenia. Some clinicians subsume a diagnosis of OCD within a hierarchical model. It is then assumed that if the psychosis is treated then the obsessive-compulsive symptoms will also improve. However, when psychotic symptoms are controlled then the symptoms of OCD often become more prominent and should be treated in their own right.

In young people, there may be a greater risk that OCD is wrongly diagnosed as a psychotic illness, especially if they present with unusual obsessions, which are mistaken for delusions. Sometimes the distress caused by bizarre and frightening obsessions leads to fear-driven behaviours, which are interpreted as behavioural manifestations of psychosis, such as shouting out loud in an attempt to cancel out thoughts. In one series of young people presenting with unusual obsessions about magically acquiring unwanted characteristics from others (termed 'transformation obsessions'), the majority had previously received an incorrect diagnosis of psychosis and had often been prescribed unnecessary antipsychotic medication (Volz 2007). Sometimes severe avoidance behaviour and selfneglect in a person with OCD appears similar to negative symptoms of schizophrenia and the diagnosis of OCD is overlooked.

There are extremely rare cases of comorbid command hallucination in OCD (usually in the context of trauma and an emotionally unstable personality disorder), which can potentially lead to violence. Such hallucinations (which are usually ego-syntonic) can be confused as being related to the obsession but are in fact distinct processes. The assessment should focus on the emotional stability and propensity to shift into a psychotic state and the contexts in which it occurs.

\section{Antisocial personality disorder}

In extremely rare cases, OCD exists with comorbid antisocial personality disorder and a history of crime and violence, in which some of the intrusive urges are ego-dystonic in some domains (e.g. contamination) but not in others (e.g. violent impulses). Alternatively, some of the intrusions may be ego-syntonic (e.g. people who the person believes 'deserve' to be harmed) and ego-dystonic at other times (e.g. causing harm to loved ones by not being careful enough).

\section{Assessing secondary risk of harm to family members}

Family members respond to OCD in many different ways. They may accommodate an individual's 
avoidance and compulsions, either allowing them to engage in them or becoming involved in them and so reinforcing them. In this regard, the person with OCD may be bullying some members of the family to comply with their compulsions. Alternatively, the family may be overprotective and seek to minimise distress by allowing the person with OCD to reduce their sphere of activities. They can also become aggressive or sarcastic and this interpersonal conflict can exacerbate the problem. Others may deny or minimise the problem or avoid the person with OCD as much as possible. Sometimes the behaviours associated with the disorder may cause family members to become restricted in their activities (e.g. using the bathroom) or their freedom to use certain rooms in the home (e.g. because of hoarding) and they may become reluctant to invite visitors.

When a carer or family member becomes completely involved in the management of a person's OCD, the risks of exhaustion, distress and depression associated with the burden of care may also need to be assessed. This is a particular problem in the older parents of adults with chronic OCD. The individual is able to function only as a result of complete devotion on the part of their parents, whose 'retirement' gradually slips away. The parents, apart from their own self-sacrifice, fear (accurately) that on their death their son or daughter will be unable to cope. However, the son or daughter is so focused on day-to-day obsessions that they neither see their parents' distress nor the likely consequences of continued dependence or failure to seek treatment.

Frequently, family members' coping mechanisms differ, leading to further discord when they disagree over the best way of dealing with the situation. As noted above, when someone in the family who usually accommodates a ritual or avoidance refuses to do so or accidentally interferes in a compulsion, an individual with OCD may become severely anxious and aggressive. The problem more often occurs in adolescents or individuals with Asperger syndrome, who may react impulsively or lack empathy. Such incidents are uncommon, but if the behaviour were to occur repeatedly and there is a risk of significant harm, then it would become of greater concern and may prompt the individual or family to seek help. A collateral history, sometimes without the person with OCD being present, may therefore be important.

\section{Action if risk is identified}

If family members are at risk, then a decision will need to be made about admitting the individual with OCD to hospital or finding alternative accommodation. The ideal goal is to help family members to be emotionally supportive without accommodating or excessively controlling the person with OCD. At times families may decide to compromise as they reduce their involvement. Changes may take time to implement if members of the family have been enmeshed in the rituals for a long time. Equally, the person with OCD will need to accept that they cannot expect others to comply with their demands or to live with such behaviour. If they feel threatened, family members should be asked to call the police or to seek protection through the courts. Ideally, family members work with sufferers to support them in the process of change, rather than supporting continued obsessions and compulsions.

\section{Assessing secondary risk of harm to young children}

A bigger problem may exist when an adult with OCD has charge of young children who are forced to accommodate the disorder. Sometimes the risk of physical harm is minimal, for example overzealousness about hand-cleaning, insistence that a child is bathed when they come into the home or refusal to allow outside play. However, in some situations, the behaviour of a person with OCD may become emotionally or physically abusive (e.g. using disinfectants such as bleach to clean the child; never allowing the child to play with other children) even though their desire is to be a good parent. In such cases, the individual is usually extremely caring towards their children and strongly values being a good parent. Probably the greatest risk to children is emotional neglect arising from the parent's preoccupation. An adult in the grip of OCD can be insensitive to the needs of a child for stimulation and emotional warmth. In hoarding, the obsessional problem takes over the child's environment; in extreme cases, the child may have to sleep in a small space cleared in the hoarder's possessions, or the house may have become a hazard as a result of the behaviour. At the other extreme, normal amenities, including heating and lighting, might be denied to the child because of the adult's preoccupations. A collateral history is an important basis on which to discuss with the parent or carers who do not have OCD the possible risks to the child.

\section{Involving child protection services}

A key issue in assessment is the discrepancy between the degree of harm that the person with OCD believes they are causing to their child and that which the child, another family member or the professional believes is being caused. Ultimately 
it may be appropriate to notify Social Services, especially when the person lacks insight into the degree of harm being caused to the child and is refusing to engage in treatment. Ensuring proper care of the children can be a helpful motivator, but not when couched in terms of a threat. The prospect of involving care agencies or the act itself may inhibit the patient from engaging properly with treatment. If the treating clinician can see no hope of sufficiently speedy change (i.e. within the timescale likely to avert serious harm to the children), referral to social services needs to be discussed. If possible, referral should be made by someone not directly involved in the treatment in order to minimise negative effects on the therapeutic alliance. It is to be hoped that treatment will continue in parallel with proceedings related to child protection.

\section{Siblings}

Finally, especially with children and adolescents with OCD, it is important to consider the risk to siblings. There are circumstances where siblings sharing a room with a child with OCD may became very distressed, for example, by the child who is up all night ritualising, preventing them sleeping, and so affecting school performance and other aspects of functioning. Aggression can be especially marked between a child suffering from OCD and their siblings. Sometimes a brother or sister becomes incorporated into obsessions, the child with OCD believing they are 'contaminated' by them, and avoiding all contact with them or even with anything they have touched. Sometimes, siblings without OCD learn quickly to identify the triggers for their affected sibling's fears and purposely taunt them by forcing exposure to a feared stimulus such as an object believed to be dirty. Although encouraging the whole family to help with exposure tasks as part of therapy can be helpful, this needs to be done with the patient's explicit permission and built into a graded exposure programme rather than being imposed. Purposeful or even unintentional teasing by a sibling can cause aggressive outbursts from the child with OCD.

\section{Assessing secondary risk in people with poor insight}

Chronic, severe OCD may leave individuals housebound and severely disabled, but if they lack insight into their condition they may not be ready to change. Patients may have developed a reverse sleep pattern, a history of self-neglect and excessive dependence on their parents for several years. They may be unable to attend out-patient clinics and their physical health may have been compromised. Typically the parents are at the end of their tether, having supported their child for many years and may appeal to their general practitioner and local mental health services for help. They might be told that because their child is not suffering from a 'severe mental illness', or is not going to take their own life or harm anyone in the community and is not psychotic, an outpatient appointment is sufficient: 'If they don't want to come to such an appointment then that is their choice'. Such patients may pose dilemmas for mental health professionals and be difficult to engage, but severe mental disorder should be characterised by the degree of handicap not the diagnosis. If a local service is unable to assist or does not have the expertise then the person should be referred to a specialist service for OCD.

\section{Access to and engagement in treatment}

Domiciliary visits may be needed for individuals with poor insight or those who have become housebound. They might be encouraged to engage with a cognitive-behavioural model of OCD, offered medication and helped to follow their important directions in life despite their disorder. The family may also need help in working as a team to set progressive boundaries around their involvement. People who cannot be treated as out-patients or on domiciliary visits may find admission to a specialist residential unit with frequent CBT more helpful.

Involuntary admission on an acute ward should be avoided if at all possible. Cognitive-behavioural therapy without the patient's active involvement is almost certain to fail, so involuntary admission is only used when any secondary risks reach serious proportions or there is a need to break a cycle of involvement by a relative. The Mental Health Act can theoretically be used for a trial of medication because of a serious risk to the patient or a need to remove the patient from their family or home environment. When patients are admitted to an acute ward, little is usually gained by staff trying to control compulsions, although boundaries may be needed for the practical running of a ward (e.g. the number of paper rolls or plastic gloves used or length of time taken in a communal shower).

Cognitive-behavioural therapy has to be done on a voluntary basis but if a patient with OCD has to be detained under the Mental Health Act, then it would be good practice for an experienced therapist to try to start the process of engagement and a formulation of the factors that are maintaining the problem. If the process is successful then CBT can be continued on a voluntary or out-patient basis. 


\begin{tabular}{|lllll|}
\hline \multicolumn{6}{|c|}{ MCQ answers } \\
1 & 2 & 3 & 4 & 5 \\
af & af & af & af & af \\
bt & bt & bf & bf & bt \\
cf & cf & cf & cf & cf \\
df & df & df & dt & df \\
ef & ef & et & ef & ef \\
\hline
\end{tabular}

Sometimes transfer to a specialist unit in OCD and finding alternative accommodation may assist in breaking a cycle which became established in the family environment.

\section{When living at home is no longer an option}

If a young person is unlikely to be able to manage on their own and the parents can no longer cope, then they should be advised on how to request that their child be rehoused or otherwise accommodated. Seeking rehousing may motivate someone with OCD to change if they feel unable to cope without family support. The NICE (2005) guidelines also recognise that some people with chronic OCD and who have never developed an adequate level of functioning may need supportive accommodation in addition to therapy.

\section{Assessing risk in therapy}

Successful CBT often involves exposure or behavioural experiments that might be regarded by some health practitioners as having a health risk or as morally unacceptable. Examples include putting one's hand down a toilet bowl; playing with a Ouija board or trying to wish death on a loved one. These tasks need to be viewed in the context of the patient's obsessions. Furthermore, the tiny risk from tasks such as exposure to contaminants needs to be weighed against the much larger mental health risks and handicap from not doing such tasks. When patients leave specialist units it is essential that professionals liaise so that patients are supported in repeating such tasks and are not undermined by well-meaning health professionals less familiar with OCD and treatment with CBT.

\section{Services for people with OCD}

The NICE guidelines (National Collaborating Centre for Mental Health 2005: p. 13) state that:

Each PCT [primary care trust], mental healthcare trust and children's trust that provides mental health services should have access to a specialist OCD/BDD [body-dysmorphic disorder] multidisciplinary team offering age-appropriate care. This team would perform the following functions: increase the skills of mental health professionals in the assessment and evidence-based treatment of people with OCD or BDD, provide high-quality advice, understand family and developmental needs, and, when appropriate, conduct expert assessment and specialist cognitivebehavioural and pharmacological treatment.

We would recommend that each mental health trust should as a minimum have a team with at least one cognitive-behavioural therapist and one consultant psychiatrist experienced in OCD who are a formally configured service. They can conduct the more difficult risk assessments, try to engage and treat patients with poor insight and decide which patients should be referred onwards to specialist centres for more intensive treatments or therapists more experienced in OCD.

\section{References}

Baron-Cohen S (2001) Theory of mind and autism: a review. In International Review of Research in Mental Retardation: Autism (ed LM Glidden): 169-84. Academic Press.

Dimidjian S, Hollon SD, Dobson KS, et al (2006) Randomized trial of behavioral activation, cognitive therapy, and antidepressant medication in the acute treatment of adults with major depression. Journal of Consulting and Clinical Psychology, 74: 658-70.

Freeman JB, Leonard HL (2000) Sexual obsessions in obsessivecompulsive disorder. Journal of American Academic Child and Adolescent Psychiatry, 39: 141-2.

Geller DA (2006) Obsessive-compulsive and spectrum disorders in children and adolescents. Psychiatric Clinics of North America; 29: 353-70.

Gordon H, Grubin D (2004) Psychiatric aspects of the assessment and treatment of sex offenders. Advances in Psychiatric Treatment, 10: 73-80.

Healy N, Fitzpatrick C, Fitzgerald E (1991) Clinical note: childhood neurotic disorders with a sexual content need not imply child sexual abuse. Journal of Child Psychology and Psychiatry, 32: 857-63.

Hoehn-Saric R, Ninan P, Black DW, et al (2000) Multicenter double-blind comparison of sertraline and desipramine for concurrent obsessivecompulsive and major depressive disorders. Archives of General Psychiatry, 57: 76-82.

Maina G, Albert U, Bogetto F, et al (1999) Recent life events and obsessive-compulsive disorder (OCD): the role of pregnancy/delivery. Psychiatry Research; 89: 49-58.

Mancini C, Van Ameringen M, Farvolden P(2002) Does SSRI augmentation with antidepressants that influence noradrenergic function resolve depression in obsessive-compulsive disorder? Journal of Affective Disorders; 68: 59-65.

Marshall WL, Langton CM (2005) Unwanted thoughts and fantasies experienced by sexual offenders. In Intrusive Thoughts in Clinical Disorders: Theory, Research, and Treatment (ed DA Clark): 199-225. Guilford Press.

National Collaborating Centre for Mental Health (2005) ObsessiveCompulsive Disorder: Core Interventions in the Treatment of ObsessiveCompulsive Disorder and Body Dysmorphic Disorder. Clinical Guideline 31. NICE.

Rachman SJ, de Silva P (1978) Abnormal and normal obsessions. Behaviour Research and Therapy, 16: 233-48.

Rachman S (1997) A cognitive theory of obsessions. Behavior Research and Therapy, 35: 793-802.

Salkovskis P (1985) Obsessional-compulsive problems: a cognitivebehavioural analysis. Behaviour Research and Therapy, 23: 571-83.

Salkovskis P, Kirk J (2008) Obsessional disorders. In Cognitive Behaviour Therapy for Psychiatric Problems: A Practical Guide leds K Hawton, PM Salkovskis, J Kirk, et al): 129-68. Oxford University Press.

Sichel DA, Choen LS, Dimmock JA, et al (1993) Postpartum obsessivecompulsive disorder: a case series. Journal of Clinical Psychiatry, 54: $156-9$.

Storch EA, Geffken GR, Merlo LJ, et al (2007) Family accommodation in pediatric obsessive-compulsive disorder. Journal of Clinical Child and Adolescent Psychology, 36: 207-16.

Torres A, Prince M, Bebbington P, et al (2006) Obsessive compulsive disorder: prevalence, comorbidity, impact, and help-seeking in the British National Psychiatric morbidity study of 2000. American Journal of Psychiatry, 163: 1978-85.

Veale D (2002) Over-valued ideas: a conceptual analysis. Behaviour Research and Therapy, 40: 383-400. 
Veale D (2007) Cognitive-behavioural therapy for obsessive-compulsive disorder. Advances in Psychiatric Treatment, 13: 438-46.

Veale D (2008) Behavioural activation for depression. Advances in Psychiatric Treatment, 14: 29-36.

Veale D, Willson R (2005) Overcoming Obsessive Compulsive Disorder. Robinson.
Volz C, Heyman I (2007) Transformation obsession in young people with obsessive-compulsive disorder (OCD). Journal of the American Academy of Child and Adolescent Psychiatry, 46: 766-72.

Warwick HM, Salkovskis PM (1990) Unwanted erections in obsessive-compulsive disorder. British Journal of Psychiatry, 157: 919-21.
MCOs

1 Intrusive sexual thoughts about children:

a are always a sign of a paedophilia

b occur in the normal population

c should necessitate immediate reporting to social services

$\mathrm{d}$ are a sign that a person is bad and disgusting

e can be suppressed if the person tries hard enough.

2 People with OCD:

a are never violent or aggressive

b may have intrusive violent thoughts which are ego-dystonic and repugnant

c never act on urges of self-harm

$d$ have a higher risk of acting on their intrusive violent thoughts

e are at a lower risk of taking their own life.
3 When depression occurs secondary to OCD:

a the patient usually describes their depression as their biggest problem

b the symptoms of depression usually develop before the onset of OCD

c noradrenergic reuptake inhibitors should be used before serotonin reuptake inhibitors for treating depression in OCD

$d$ the symptoms of depression can only be treated with antidepressant medication

e the symptoms of depression are usually overcome when OCD is treated.

4 In people with OCD who are housebound and have little insight:

a admission under Mental Health Act should always be used

b an out-patient appointment should be offered and it is up to them if they want to attend c the parents are the main problem since they collude with the patient

$d$ every effort should be made to engage the person in treatment on domiciliary visits

e rehousing away from carers is unlikely to motivate a person with OCD to change.

5 Children and adolescents with OCD:

a should always be referred to social services if they disclose sexual obsessions

b should always be invited to have their parents involved in their treatment

c are more likely to be developing a homosexual orientation if they have sexual obsessions

$\mathrm{d}$ will not behave aggressively unless they have a comorbid diagnosis in addition to OCD

e may have more insight into the unnecessary nature of obsessions and compulsions.

\section{'Amber innocent ' by Joan Adeney Easdale}

\section{Selected by Femi Oyebode}

The sun was sinking.

In an upstairs window

A tailor's dummy underwent transfiguration.

A crimson tape-measure crossed the bust

As an order, such as queens wear,

And her diamonds were scintillating dust.

Paper-covered books curled,

A needle slid towards the scissors on the sill,

Shadows unfurled

Like rolls of dark crêpe across the counter.
For so things happen when the tailor turns the key And goes away, and no one's there to see. None can witness when a change is wrought Till afterwards. Few have heard The last tick before the clock stops short Or seen the crack appear upon the ceiling.

\section{POEM}

